\title{
Helium droplet assisted synthesis of plasmonic Ag@ZnO core@shell nanoparticles
}

\author{
Alexander Schiffmann ${ }^{1}(\bowtie)$, Thomas Jauk ${ }^{1}$, Daniel $\mathrm{Knez}^{2}$, Harald Fitzek ${ }^{2}$, Ferdinand Hofer ${ }^{2}$, Florian Lackner ${ }^{1}$, \\ and Wolfgang E. Ernst ${ }^{1}(\square)$ \\ ${ }^{1}$ Institute of Experimental Physics, Graz University of Technology, A-8010 Graz, Austria \\ ${ }^{2}$ Institute of Electron Microscopy and Nanoanalysis \& Graz Centre for Electron Microscopy, Graz University of Technology, A-8010 Graz, \\ Austria
}

(C) The Author(s) 2020

Received: 21 February 2020 / Revised: 26 June 2020 / Accepted: 27 June 2020

\begin{abstract}
Plasmonic Ag@ZnO core@shell nanoparticles are formed by synthesis inside helium droplets with subsequent deposition and controlled oxidation. The particle size and shape can be controlled from spherical sub-10 nm particles to larger elongated structures. An advantage of the method is the complete absence of solvents, precursors, and other chemical agents. The obtained particle morphology and elemental composition have been analyzed by scanning transmission electron microscopy (STEM) and energy dispersive X-ray spectroscopy (EDS). The results reveal that the produced particles form a closed and homogeneous $\mathrm{ZnO}$ layer around a $2-3 \mathrm{~nm} \mathrm{Ag}$ core with a uniform thickness of $(1.33 \pm 0.15) \mathrm{nm}$ and $(1.63 \pm 0.31) \mathrm{nm}$ for spherical and wire-like particles, respectively. The results are supported by ultraviolet photoelectron spectroscopy (UPS), which indicates a fully oxidized shell layer for the particles studied by STEM. The plasmonic properties of the produced spherical Ag@ ZnO core@shell particles are investigated by two-photon photoelectron (2PPE) spectroscopy. Upon excitation of the localized surface plasmon resonance in $\mathrm{Ag}$ at around $3 \mathrm{eV}$, plasmonic enhancement leads to the liberation of electrons with high kinetic energy. This is observed for both $\mathrm{Ag}$ and $\mathrm{Ag} @ \mathrm{ZnO}$ particles, showing that even if a Ag cluster is covered by the $\mathrm{ZnO}$ layer, a plasmonic enhancement can be observed by photoelectron spectroscopy.
\end{abstract}

\section{KEYWORDS}

nanoparticle, helium droplet, plasmonics, photoelectrons

\section{Introduction}

Zinc oxide has become one of the most popular materials for applications in the fields of photocatalysis and optoelectronics due to its intriguing properties. $\mathrm{ZnO}$ is a non toxic n-type semiconductor with a wide direct band gap of $3.37 \mathrm{eV}$, very similar to $\mathrm{TiO}_{2}$ but with a higher absorption efficiency under solar irradiation and a large exciton binding energy of about $60 \mathrm{meV}$ [1]. As a photocatalyst it shows high potential in degradation processes of organic pollutants via the generation of reactive oxygen species and also exhibits antibacterial properties, especially when scaled down to the nano regime [2]. Due to its photoconductivity and photoluminescence characteristics, applications of $\mathrm{ZnO}$ in optoelectronics are possible, for example, in ultraviolet (UV) detectors, solar cells, lasers, and various sensing devices $[3,4]$.

In recent years, miniaturization and engineering of material properties on the nanometer scale have strongly influenced the research on $\mathrm{ZnO}$. Starting with needles and other structures in the micrometer size regime [5], by now the synthesis of $\mathrm{ZnO}$ nanoparticles is an important branch of research. An increasing amount of one-, two-, and three-dimensional structures have been synthesized in shapes ranging from nanoparticles, -rods, and -ribbons over nanoplates to exotic flower or snowflake structures [6]. The formation of $\mathrm{ZnO}$ based composite materials opens up further possibilities for the tuning of properties, which permit the addition of new functionalities and, consequently, the doping of $\mathrm{ZnO}$ receives increasing attention. Different material groups can be used as dopants, for example, to produce p-type $\mathrm{ZnO}$, enable applications in spintronics, or to improve optical and electrical properties [7]. A modification with Ag can significantly enhance the photocatalytic activity of $\mathrm{ZnO}$ [8] because the electron-hole pair concentration is increased by the strong local fields induced by the excitation of the localized surface plasmon in Ag [9].

Most of the synthesis routes for pure or doped nanosized $\mathrm{ZnO}$ have in common that they are based on multi-step chemical processes involving solvents or precursors. Though those techniques are well controlled and documented, it is possible that residues from chemical agents or surfactants remain at the particle surface or even at the core-shell interface.

In this study, the helium nanodroplet synthesis approach is employed as a pristine and facile technique for the synthesis of Ag@ZnO core@shell nanoparticles. This process bears the advantage of dispensing no chemicals, additives, or other additional agents besides the high purity metals, which are doped into the superfluid helium droplets where they agglomerate to nanoparticles. Since the whole procedure is conducted under ultra-high vacuum (UHV) conditions, a contamination of the 
sample and other influences from environmental sources are excluded. Due to the dependence of the particle size and shape solely on a few well defined and accurately adjustable experimental parameters the approach allows for a high reproducibility. The range of accessible shapes spans from smaller spherical to longer wire-like nanoparticles, as shown in the course of this study. The obtained particle size and shape depends on the helium droplet source conditions, with which the helium droplet size can be controlled [10]. Small helium droplets lead to the formation of spherical nanoparticles, typically with a diameter below $5 \mathrm{~nm}$. As a consequence of their superfluidity, large droplets host quantum vortices [11], along which dopants preferentially agglomerate enabling the formation of wire-like nanoparticles. Even though the scalability of the production is limited because only a few $100 \mathrm{ng}$ per hour can be produced, the method allows for an investigation of fundamental particle properties. Furthermore, for future experiments the synthesis of core@shell species with a wide range of material combinations is accessible with one experimental setup simply by exchanging the dopant metal sources. Electron microscopy has been used to probe the size distribution, morphology, and composition of the deposited nanoparticles, revealing that the Ag cores are covered by a uniformly shaped wurtzite $\mathrm{ZnO}$ shell. Further investigations on the oxidation state of the $\mathrm{ZnO}$ shell have been conducted by ultraviolet photoelectron spectroscopy and two-photon photoelectron spectroscopy was employed to demonstrate the plasmonic activity of the $\mathrm{ZnO}$ covered Ag core.

\section{Experimental}

\subsection{Nanoparticle synthesis}

The ZnO and Ag@ZnO core@shell nanoparticles investigated in this work were produced utilizing the helium nanodroplet approach. This technique employs superfluid helium droplets $\left(\mathrm{He}_{\mathrm{N}}\right)$ as an inert environment for the synthesis of nanosized metallic particles [12-14]. Details about the experimental design of the $\mathrm{He}_{\mathrm{N}}$ apparatus can be found in Ref. [15]. In summary, a closed cycle refrigeration unit (Sumitomo RDK-408D2) is used to cool gaseous helium (99.9999\% purity) at a stagnation pressure of 20-60 bar down to only a few Kelvin. The helium is then adiabatically expanded into high vacuum $\left(\sim 10^{-5} \mathrm{mbar}\right)$ through a $5 \mu \mathrm{m}$ diameter nozzle resulting in a beam of superfluid $\mathrm{He}_{\mathrm{N}}$. The internal temperature of the $\mathrm{He}_{\mathrm{N}}$, thereby, drops to $0.37 \mathrm{~K}[16,17]$. The droplet sizes can be controlled by the temperature and the stagnation pressure of the helium gas. For this work a setup with a pressure of 20 bar at $8 \mathrm{~K}$ was used for the synthesis of spherical nanoparticles resulting in a He droplet diameter of about $70 \mathrm{~nm}$ with a mean number of about $5 \times 10^{6} \mathrm{He}$ atoms [10]. For the larger wire-like particles, the parameters were changed to 20 bar and $5 \mathrm{~K}$ leading to $\mathrm{He}_{\mathrm{N}}$ diameters of around $1 \mu \mathrm{m}$, consisting of about $2 \times 10^{10} \mathrm{He}$ atoms [10]. Subsequently, the collimated $\mathrm{He}_{\mathrm{N}}$ beam passes through another chamber $\left(\sim 10^{-7} \mathrm{mbar}\right)$ where resistively heated tungsten baskets coated with aluminium oxide are used as pickup cells for the evaporation of high purity metals. While passing through the metal vapor, the $\mathrm{He}_{\mathrm{N}}$ are doped with the evaporated atoms.

The superfluidity of the $\mathrm{He}_{\mathrm{N}}$ matrix enables an unobstructed and frictionless roaming of dopant atoms. Upon statistical collisions within the $\mathrm{He}_{\mathrm{N}}$, the dopants accumulate and start to form clusters $[18,19]$. Initially, these clusters are grown as multicenters inside a droplet before they further agglomerate to larger structures. The final size of the nanoparticles depends on the doping rate and on the initial $\mathrm{He}_{\mathrm{N}}$ size, since both affect the average time between collisions and, thereby, the growth rate [20]. The synthesis of wire-like nanoparticles is enabled by the presence of vortices in larger He droplets with diameters of several hundred nanometers. These vortices form due to the angular momentum acquired by the superfluid droplets during the expansion of the helium through the nozzle into vacuum $[11,21,22]$. The dopant metal atoms and grown clusters inside the droplets can agglomerate along such vortices due to a pressure gradient directed towards the vortex core [23]. By adjusting the size of the droplets accordingly and by doping enough material into them, elongated wire-like structures can be formed along the vortices [20,24, 25]. As stated above for the two employed parameter settings in this experiment, a change in the helium source conditions directly influences the size of the $\mathrm{He}_{\mathrm{N}}$ and allows for a facile switching between the different nanoparticle types. It should be noted that by decreasing the source temperature not only the droplet size is increased, also the total $\mathrm{He}_{\mathrm{N}}$ flux and the deposition rate are enhanced. During agglomeration of atoms and clusters, the released binding energy is dissipated by the evaporation of $\mathrm{He}$ atoms from the droplet. The attenuation of the $\mathrm{He}_{\mathrm{N}}$ beam due to the decrease in droplet size caused by this process is measured in order to monitor the particle growth process [20]. The synthesis of core@shell particles is enabled by the subsequent doping of two different metals in consecutive pickup cells $[26,27]$. Before entering the second pickup cell holding the shell material, the particle cores have already formed with the metal provided in the first cell. The metal doped to the droplets in the second pickup cell forms a shell layer around the core [28]. The dopant vapor pressure in each cell and, thereby, the metal atom doping rate and the nanoparticle growth are directly controlled by the temperature of the resistively heated pickup cells. Since the pickup cells are separately controlled, the amount of doped core and shell material can be adjusted independently. This allows for a tailoring of the core diameter and the shell thickness of the synthesized nanoparticles within a certain range, as has been shown for Ag@Au [29] and Fe@Au [30]. For this work, the pickup cells were loaded with $\mathrm{Ag}$ and $\mathrm{Zn}$, enabling the formation of pure Ag, Zn and Ag@Zn core@shell particles inside the helium droplets.

After leaving the pickup region, the $\mathrm{He}_{\mathrm{N}}$ beam reaches a third chamber at UHV conditions $\left(\sim 5 \times 10^{-10} \mathrm{mbar}\right)$, where the deposition rate is monitored by termination of the beam on a quartz crystal microbalance. For deposition of the nanoparticles the substrates are inserted into the beam path with a wobblestick transfer system coupled to a vacuum suitcase (Ferrovac GmbH, NexGeneration UHV Suitcase). The suitcase allows for the storage and transport of the samples at UHV conditions $\left(\sim 1 \times 10^{-10} \mathrm{mbar}\right)$. Due to the different employed analysis methods, support grids for scanning transmission electron microscopy (STEM) (Ted Pella Inc., Ultra-thin Carbon Film on Lacey Carbon Support Film, 300 mesh, Au) as well as glass coverslips coated with indium tin oxide (ITO) (spi Supplies, $30-60 \Omega, 18 \times 18 \mathrm{~mm}$, No. 1) were used as substrates. As the $\mathrm{He}_{\mathrm{N}}$ beam is terminated at the substrate, the particles are cushioned by the surrounding helium and, therefore, keep their shape upon deposition under soft landing conditions [31, 32] as observed by atomic resolution electron tomography [33]. To start the oxidation of the $\mathrm{Zn}$ within the nanoparticles, the vacuum chamber with all the decorated substrates inside, was flushed with pure oxygen up to a pressure of $1 \times 10^{-6} \mathrm{mbar}$ for $1 \mathrm{~h}$. A single carbon substrate has been decorated with both fabricated species, i.e. the spherical and wire-like nanoparticles, for the STEM experiments. During transport to the STEM, this sample was exposed to ambient air for about 1 hour. The spherical particles deposited on the ITO substrates for the 
UPS and 2PPE experiments have initially not been exposed to ambient air due to the use of the vacuum suitcase for the transport. Only later, in the course of the experiment, these samples have been deliberately exposed to air in order to achieve complete oxidation.

\subsection{Scanning transmission electron microscopy}

A detailed analysis of the nanoparticle structure and composition was conducted with a probe-corrected FEI Titan ${ }^{3}$ 60-300 STEM with an X-FEG field emission source operated at a beam energy of $300 \mathrm{keV}$. The STEM system is equipped with an FEI Super-X four-quadrant detector for energy dispersive $\mathrm{X}$-ray spectroscopy (EDS). For the acquisition of data, the number of channels was set to 1,024 with a dispersion of $10 \mathrm{eV}$ per channel and an exposure time of $100 \mathrm{~ms}$ was used. For high-angle annular dark-field (HAADF) images the dwell time per pixel was set to $2.4 \mu \mathrm{s}$, while the size of the spectrum images (SI) was adjusted for each individual acquisition.

\subsection{Photoemission electron spectroscopy}

Photoelectron spectroscopy was conducted with a NanoESCA (FOCUS GmbH \& Scienta Omicron) energy filtered photoemission electron microscope (EF-PEEM) at UHV conditions $\left(\sim 3 \times 10^{-11} \mathrm{mbar}\right)$. A helium discharge lamp (HIS) operated at $21.2 \mathrm{eV}$ and the second harmonic of an Indigo-S Ti:Sapphire laser system (Coherent) tuned to $3.02 \mathrm{eV}(410 \mathrm{~nm})$ were used as photon sources for the acquisition of ultraviolet photoelectron spectra (UPS) and two-photon photoelectron (2PPE) spectra, respectively. The pulse duration of the laser was $30 \mathrm{~ns}$ with a repetition rate of $5 \mathrm{kHz}$. The spot size of the HIS on the sample surface was about $330 \mu \mathrm{m}$, while the laser was focused to a spot size of about $50 \mu \mathrm{m}$ (full width at half maximum) at a laser power of $0.5 \mathrm{~mW}$. With the pass energy for the energy filter set to $50 \mathrm{eV}$ and an entrance slit of $1 \mathrm{~mm}$, an energy resolution of $200 \mathrm{meV}$ was reached. An extractor field of $12 \mathrm{kV}$ was applied with a sample distance of $1.63 \mathrm{~mm}$. The energy filtered photoelectrons were collected and counted with a channeltron (CT), the measurement area was restricted to a spot size with $(7 \pm 1) \mu \mathrm{m}$ diameter using an iris aperture.

\section{Results and discussion}

\subsection{Nanoparticle structure and morphology}

For STEM analysis, an amorphous carbon TEM grid was decorated with two different types of nanoparticles in order to give an example of the capabilities of the helium nanodroplet synthesis approach in terms of possible particle shapes. These two selected types encompass small, spherically shaped Ag@ZnO core@shell nanoparticles (source conditions: 20 bar and $8 \mathrm{~K}$ ) deposited for $40 \mathrm{~min}$, as well as wire-like Ag@ZnO core@shell nanoparticles (source conditions: 20 bar and $5 \mathrm{~K}$ ) deposited for $3 \mathrm{~min}$. Note that both types of nanoparticles were deposited on a single TEM grid, hence both species can be seen in the images presented in the following. During transport and transfer into the STEM, the sample was exposed to ambient conditions for about $1 \mathrm{~h}$ in total, which is enough time for the $\mathrm{Zn}$ shell to completely oxidize. This finding is in agreement with previous studies, which found that an oxide layer with a thickness up to $3 \mathrm{~nm}$ is formed directly upon exposure to air [34].

Figure 1(a) shows a selected HAADF image in which the two particle types can be identified. The bright Ag cores within the particles can be clearly distinguished from the surrounding $\mathrm{ZnO}$ since the HAADF image intensity is proportional to the square of the atomic number $\left(Z^{2}\right)$ [35]. It can be seen that the synthesis route based on the formation of
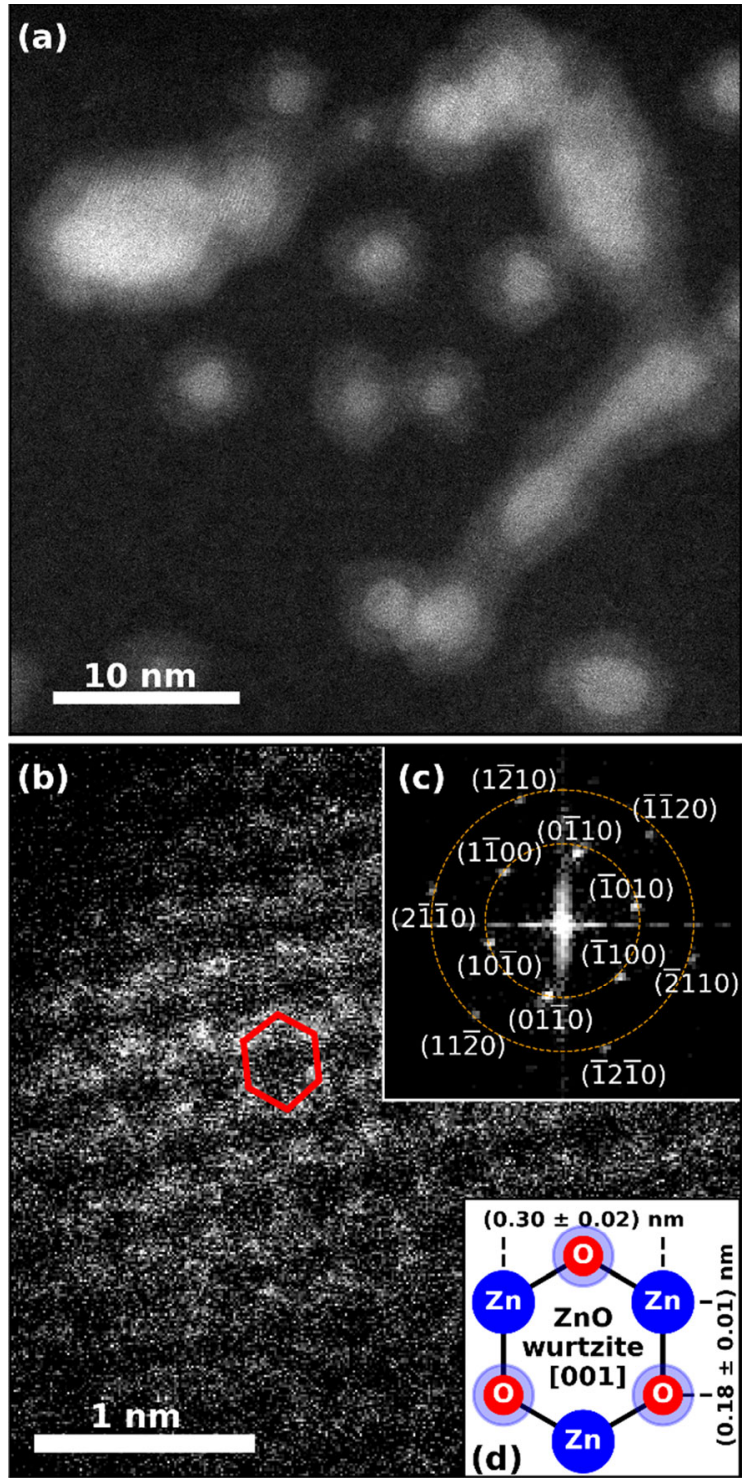

Figure 1 (a) STEM HAADF image of the synthesized Ag@ZnO nanosized particles. Spherically shaped as well as wire-like structures can be identified. The core@shell structure is clearly observable as silver cores appear brighter than zinc oxide shells. The smaller spherical particles and the elongated larger particles were created at different helium droplet beam conditions and were deposited on the same substrate subsequently (cf. chapter 2.1). (b) Detailed HAADF image showing ZnO along the [001] axis with the hexagonal structure highlighted in red for a selected unit cell. Inset plot (c) shows the fast Fourier transform (FFT) of the STEM image, yielding periodicities of $(0.30 \pm 0.02) \mathrm{nm}$ and $(0.18 \pm 0.01) \mathrm{nm}$ marked by the dashed orange circles. The sixfold symmetry of the FFT pattern is clearly observable and all visible features are assigned. The schematic in inset plot (d) explains the origin of the two observed lengths within the $2 \mathrm{D}$ projection of the hexagonal unit cell of wurtzite $\mathrm{ZnO}$ in [001] direction.

Ag@Zn particles and subsequent oxidation of the $\mathrm{Zn}$ to $\mathrm{ZnO}$ results in a very uniform shell around the core, covering the $\mathrm{Ag}$ in a $\mathrm{ZnO}$ layer of constant thickness. This is true for the smaller spherical particles as well as for the larger wire-like structures as the presented STEM image shows. The measurement and subsequent statistical analysis of a random selection of 110 spherically shaped Ag@ZnO particles yielded a mean shell thickness of (1.33 \pm 0.15$)$ nm. The larger, wire-like Ag@ZnO structures were less frequently imaged and 35 were analyzed, resulting in a mean $\mathrm{ZnO}$ shell thickness of $(1.63 \pm 0.31) \mathrm{nm}$. Further HAADF images with lower magnification are presented in Fig. S1 in the Electronic Supplementary Material (ESM), providing an overview of the particle distribution on the 
substrate. In Fig. S2 in the ESM another set of detailed highresolution HAADF images is presented to give a more thorough picture of the particle quality and the regularity of the $\mathrm{ZnO}$ shells covering the Ag cores.

A structural analysis of the uniformly grown $\mathrm{ZnO}$ within a selected particle is presented in Fig. 1 (b), which shows a detailed STEM HAADF image of the surface of a nanoparticle oriented in [001] direction. The contrast is enhanced and the observable hexagonal structure is highlighted by a red hexagon to guide the eye. The inset plot in Fig. 1(c) shows the corresponding fast Fourier transform (FFT), which allows conclusions on the crystal structure of the $\mathrm{ZnO}$ shell layer. The FFT image yields lattice distances of $(0.30 \pm 0.02) \mathrm{nm}$ and $(0.18 \pm 0.01) \mathrm{nm}$, marked by dashed orange circles. The observed pattern clearly exhibits sixfold symmetry. All visible features are assigned with the corresponding Bravais-Miller indices. The six diffraction spots on the inner circle and the six spots on the outer circle show permutation symmetry among themselves and originate from the $\{100\}$ and $\{2 \overline{1} 0\}$ planes of wurtzite $\mathrm{ZnO}$, respectively. The obtained distances are in agreement with literature bulk values for the lattice spacing of 0.2814 and $0.1625 \mathrm{~nm}$, taken from the Crystallography Open Database (COD) [36]. Deviations from the tabulated values most likely originate from the fact that the shell is only several atomic layers thick, which can lead to a lattice expansion as reported for several oxides on the nanoscale [37]. In the inset plot Fig. 1(d) the two periodically occurring lengths found in the FFT image are assigned to the two-dimensional projection of the hexagonal structure of $\mathrm{ZnO}$ in [001] direction.

Figure $\mathrm{S} 3$ in the ESM shows additional evaluated FFT images of Ag@ZnO nanoparticles in varying orientations, confirming the findings from Fig. 1. Overall, with the obvious sixfold symmetry and the lattice parameters in good agreement with literature, it is concluded that the $\mathrm{ZnO}$ shell layer prefers a wurtzite unit cell structure in case of the synthesized nanoparticles.

The chemical composition of the nanoparticles was determined by means of EDS. Figure 2(a) shows a HAADF image of several spherically shaped core@shell nanoparticles. An EDS map acquired from this area was used to create the spatially resolved elemental maps for $\mathrm{Ag}, \mathrm{Zn}$ and $\mathrm{O}$, which are shown in Figs. 2 (b)-2(d). In particular, the event counts around the $\mathrm{L}_{\alpha 1}$ peak of $\mathrm{Ag}$ at $2.984 \mathrm{keV}$, the $\mathrm{L}_{\alpha}$ peak of $\mathrm{Zn}$ at $1.012 \mathrm{keV}$, and the $\mathrm{K}_{\alpha}$ peak of $\mathrm{O}$ at $0.523 \mathrm{keV}$ were extracted from the EDS data and plotted according to their respective position in the image. It is evident that the positions of the bright core structures in the HAADF image are matching those with high abundance of Ag. The same applies for the whole particles including the shell and the respective EDS data for $\mathrm{Zn}$. The slight offsets between particle positions in the HAADF image and the EDS maps may originate from a drift of the sample or a mobilization of the nanoparticles due to the exposure to the electron beam for longer times during the acquisition. These effects can hardly be avoided since the HAADF image and EDS data are acquired in independent scans. Note that the position of oxygen is not as precise as for the metals because it is also present in the background in form of hydrocarbons on the STEM substrate. Nonetheless, there is an increased abundance of $\mathrm{O}$ in the background corrected color map at the positions where $\mathrm{Zn}$ is also found. In combination with the findings of the structural investigation shown in Fig. 1, it is concluded that the Ag cores are covered by a uniform $\mathrm{ZnO}$ shell.

\subsection{Size distribution and surface coverage}

In order to gain information about the size distribution of the deposited particles, the overall diameters and Ag core diameters

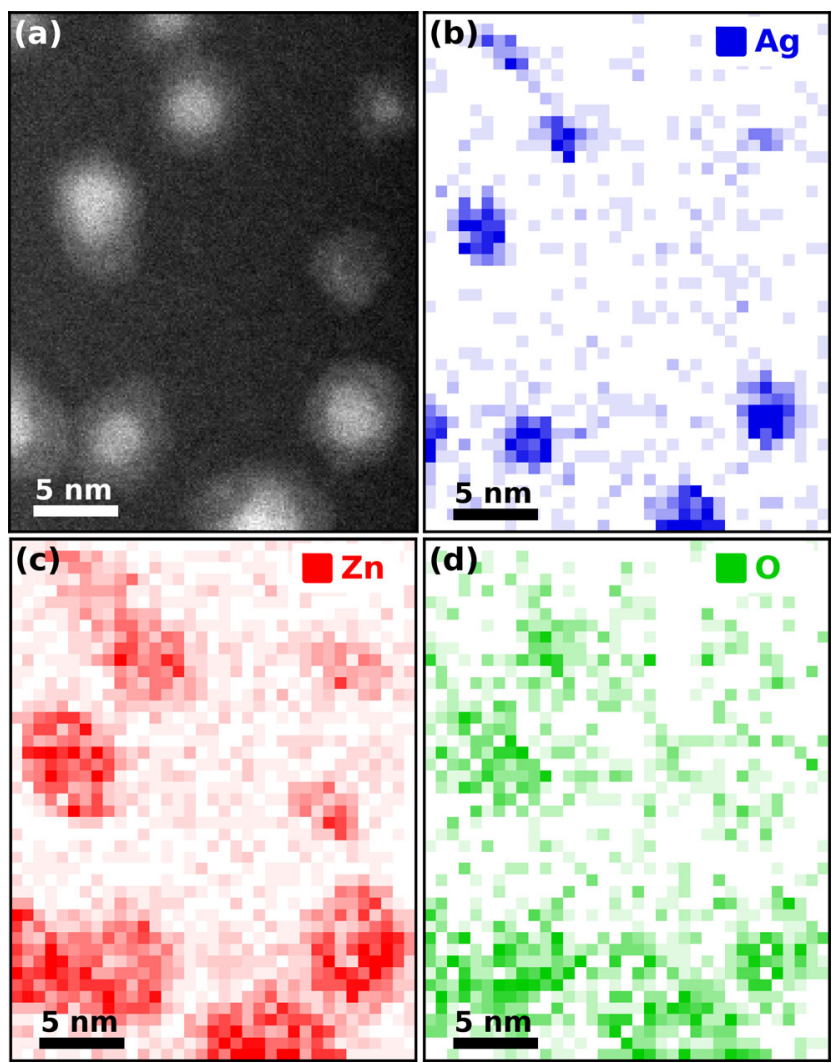

Figure 2 (a) Detailed STEM HAADF image of a selected region with spherically shaped Ag@ZnO core@shell nanoparticles. The spatially resolved EDS maps reveal the spatial distribution of (b) the Ag core material as well as (c) the $\mathrm{Zn}$ and (d) the $\mathrm{O}$ in the shell of the particles.

of 208 spherically shaped nanoparticles (synthesized at 20 bar and $8 \mathrm{~K}$ ) were individually determined from high-resolution STEM images. Examples for the STEM images can be found in Fig. S2 in the ESM. The results are shown in the histograms in Fig. 3, containing the measured overall diameters of the particles in Fig. 3(a) and the core diameters in Fig. 3(b). For both datasets the diameters are, in good approximation, normally distributed. A fit yields a mean overall particle diameter of $5.84 \mathrm{~nm}$ with a standard deviation of $0.93 \mathrm{~nm}$ and a mean core diameter of $(3.03 \pm 0.64) \mathrm{nm}$. The width of both size distributions is inherent to the $\mathrm{He}_{\mathrm{N}}$ synthesis process and originates from the broad droplet size distribution [14]. Over 100 of the wirelike nanoparticles were measured and $70 \%$ of the particles
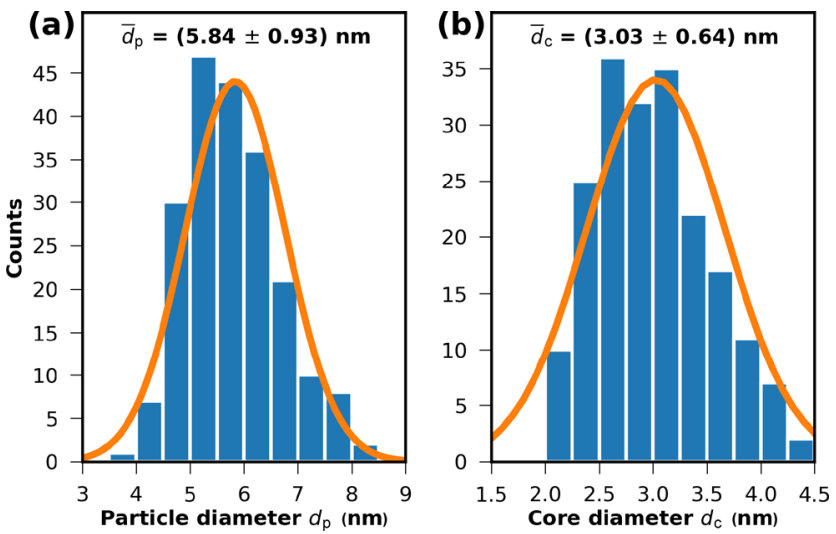

Figure 3 From high-resolution STEM images, the particle and core diameters of 208 spherically shaped Ag@ZnO nanoparticles were measured. (a) Histogram of the measured particle diameters with a fitted normal distribution yielding a mean particle diameter and standard deviation of $\bar{d}_{\mathrm{p}}=(5.84 \pm 0.93) \mathrm{nm}$. (b) Histogram of the measured Ag core diameters with the normal distribution resulting in a mean of $\bar{d}_{\mathrm{c}}=(3.03 \pm 0.64) \mathrm{nm}$. 
have a length between 20 and $50 \mathrm{~nm}$. Their diameter ranges from 6 to $8 \mathrm{~nm}$, however, can extend to over $10 \mathrm{~nm}$ for agglomerated structures. Note that the diameter can vary over the length of a single particle and that most of them are kinked or branched, which makes an exact characterization of their size difficult.

For the determination of the surface coverage for the deposited nanoparticles the overview STEM images shown in Fig. $S 1$ in the ESM were used. With the ImageJ 1.52p software package [38] it was possible to separately evaluate the spherical (deposition time: $40 \mathrm{~min}$ ) and wire-like (deposition time: $3 \mathrm{~min}$ ) particles due to a discrimination in size. This is shown in Fig. S4 in the ESM for one of the STEM images. The evaluation yields an estimation for the obtained surface coverage of about $(9.3 \pm 0.5) \%$ per hour for the spherically shaped particles and about $(110 \pm 30) \%$ per hour for the elongated structures. The difference can be explained by the different helium droplet source conditions. For colder temperatures and larger droplets the deposition rate is enhanced, but a precise control of the particle shape is no longer possible and a variety of different shapes is formed.

The determined surface coverage rate for the spherically shaped Ag@ZnO nanoparticles is used to estimate the coverage of the glass coverslips coated with ITO prepared for analysis in the NanoESCA system. All three samples (pure Zn, pure Ag, and Ag@ZnO) were produced under the same experimental conditions $(20$ bar at $8 \mathrm{~K}$ ) with a deposition duration of $5 \mathrm{~h}$ each, resulting in an estimated surface coverage of about $(45.0 \pm 2.5) \%$. Since the decorated ITO substrate cannot be analyzed by STEM, a scanning electron microscopy (SEM) overview image has been recorded, shown in Fig. S5 in the ESM. It confirms that the particles are distributed uniformly on the surface of the prepared samples, though the nanoparticles appear broadened at the given magnification due to the lower resolution of SEM.

\subsection{Ultraviolet photoelectron spectroscopy}

For the UPS and the 2PPE spectroscopy experiments with the NanoESCA system three different nanoparticle samples were prepared under the same conditions ( 20 bar at $8 \mathrm{~K}$ for $5 \mathrm{~h}$ ) to guarantee comparability: pure Ag particles, $\mathrm{ZnO}$ particles and Ag@ZnO particles. All three samples are decorated with spherically shaped particles only, since it is of importance for the interpretation of the acquired spectra that the shape is uniform and the size distribution narrow. The elongated wire-like structures, as shown in Fig. 1 (a), are kinked and exhibit a wider range of lengths and shape. Therefore, they are not further evaluated by the employed spectroscopic methods that yield results integrated over an extended surface area. Furthermore, the spherical Ag particles typically exhibit a localized surface plasmon resonance (LSPR) around $400 \mathrm{~nm}$, which is accessible by the Ti:Sapphire laser system [39]. Glass coverslides coated with ITO, which were also characterized before particle deposition, were used as substrates. The samples were transported to the NanoESCA EF-PEEM under UHV conditions.

Selected regions of the UPS spectra obtained for Ag@ZnO particles on ITO are shown in Fig. 4 as a function of the binding energy $E_{\text {bind. }}$ The spectra are normalized to the maxima of the secondary electron peak and the right part of the plot represents a zoom into the region around the Fermi cutoff. The solid lines represent the mean of five measurements at different sample spots, the $2 \sigma$ confidence intervals are represented by the shaded areas. The blue line in Fig. 4 represents the acquired UPS spectrum of the plain ITO substrate before deposition and the orange line shows the signal of the Ag@ZnO particles after the transfer to the PEEM. The

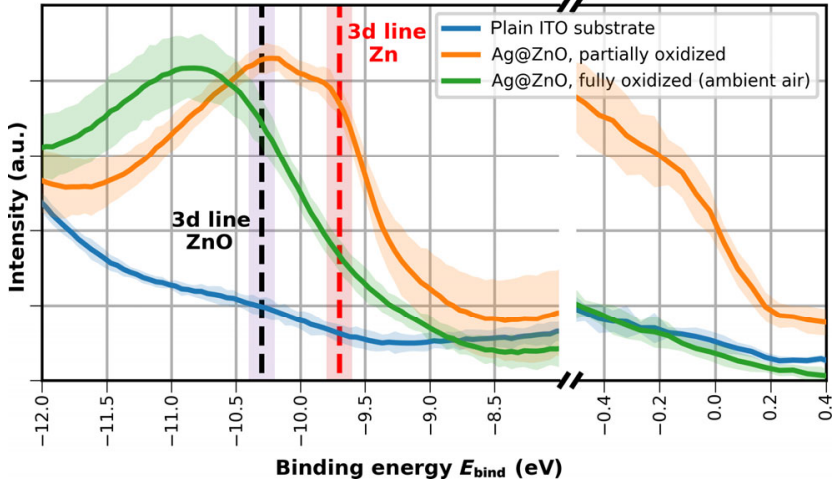

Figure 4 UPS spectra of the plain ITO substrate (blue), the partially oxidized Ag@ZnO nanoparticles after deposition (orange) and the fully oxidized Ag@ZnO particles after exposure to ambient air for $1 \mathrm{~h}$ (green). The peak positions for the double peak in the orange spectrum are marked by vertical lines in red at $-9.7 \mathrm{eV}$ and black at $-10.3 \mathrm{eV}$ and attributed to the $3 \mathrm{~d}$ level of metallic $\mathrm{Zn}$ and of oxidized $\mathrm{Zn}$, respectively. The acquired spectra are averaged over five different spots on the sample and the shaded areas represent the $2 \sigma$ confidence levels.

depicted region of interest in the left part of the diagram displays the energy range around the $3 \mathrm{~d}$ core level peak of $\mathrm{Zn}$. The orange line has a distinct and broad feature situated in this region with a double-peaked shape. A set of two Gaussians was used to evaluate the maxima of the convoluted peak structure. Thereby, the peak position at lower binding energy was found to be at $(-9.7 \pm 0.1) \mathrm{eV}$, marked by the vertical red line. The peak is attributed to the $3 \mathrm{~d}$ level of pristine $\mathrm{Zn}$. The second maximum, marked with a vertical black line, is located at $(-10.3 \pm 0.1) \mathrm{eV}$ and represents the $3 \mathrm{~d}$ level of the $\mathrm{Zn}$ atom in $\mathrm{ZnO}$. A comparison with literature confirms the drawn conclusions about the origin of the peaks, though there is a certain spread in the published data [40-42]. The peak shift due to the chemical composition allows for a qualitative assessment of the oxidation state of the $\mathrm{Zn}$ atoms in the sample [43]. To ensure that the $\mathrm{Zn}$ is fully oxidized and the particles are comparable with the ones produced for the acquisition of the STEM data, the sample was exposed to ambient air for $1 \mathrm{~h}$ and then transferred back into the UHV chamber of the PEEM. The green line in Fig. 4 shows the UPS spectrum of the Ag@ZnO particles after the exposure to ambient conditions. The double peak structure is not present anymore, instead there is a single peak centered at $(-10.8 \pm 0.2) \mathrm{eV}$, which is again attributed to the $3 \mathrm{~d}$ level of $\mathrm{Zn}$ in the oxidized state shifted to higher binding energies by $0.3-0.5 \mathrm{eV}$. This energy shift is an undesired side effect of the exposure to ambient air and the subsequent photo-hydroxylation of the sample surface driven by the extreme ultraviolet radiation during the acquisition of UPS data. The observed artifact was investigated in more detail by Gutman et al. in Ref. [44] for nano-crystalline zinc oxide thin films. Within the uncertainty of the measurement the energy shift found for the $\mathrm{ZnO}$ covered nanoparticles in this study is in good agreement with their results. However, the important conclusion is that there is no sign of any metallic $\mathrm{Zn}$ left in the shell. The inelastic mean free path of electrons in inorganic compounds, considering the $21.2 \mathrm{eV}$ photon energy provided by the HIS, is at least $\sim 1.5 \mathrm{~nm}$ [45]. As this value exceeds the shell thickness of the spherically shaped particles with $(1.33 \pm 0.15) \mathrm{nm}$, it is concluded that the shell is fully oxidized after the sample has been exposed to ambient air.

The metallic nature of the partially oxidized Ag@ZnO is also reflected by the shape of the spectrum in the region that contains information about the population at the Fermi edge 
close to $E_{\text {bind }}=0 \mathrm{eV}$. A pronounced Fermi cutoff, the spectral signature of a metal, is clearly visible for the corresponding orange spectrum. For the conductive plain ITO substrate the cutoff is barely visible and it seems to be even more suppressed for the Ag@ZnO sample, which has been exposed to air. Considering that at a surface coverage of $45 \%$ the recorded spectra correspond to a superposition of both substrate and nanoparticles, the absence of the Fermi cutoff indicates the fully oxidized, and thus semiconducting, $\mathrm{ZnO}$ as the dominant species.

Though this study aimed at the full oxidation of the $\mathrm{Zn}$ shell, there are intriguing possibilities for future experiments concerning the synthesis and formation of triple layer Ag@Zn@ZnO core@shell nanoparticles. The addition of a third layer, as already shown in literature, can potentially add new characteristics or improve existent properties of a layered nano-scaled system [46-48]. Furthermore, oxidation and reduction cycles could be applied to switch back and forth between $\mathrm{Zn}$ and $\mathrm{ZnO}$ as was already done e.g. for the $\mathrm{Ni} / \mathrm{NiO}$ system [49]. Photoelectron spectroscopy offers a reliable method to discriminate between the metallic and semiconducting state and may be employed in future experiments to explore such systems and processes.

\subsection{Two-photon photoelectron spectroscopy}

2PPE spectroscopy is employed in order to investigate the plasmonic activity of the Ag cores within the Ag@ZnO nanoparticles. The $2 \mathrm{PPE}$ experiments are also carried out on ITO substrates covered only with spherically shaped nanoparticles and the conclusions in the following are drawn by comparing 2PPE spectra recorded for pure Ag and $\mathrm{ZnO}$ to Ag@ZnO samples. Note that the species containing $\mathrm{Zn}$ were already exposed to ambient air such that the shell is fully oxidized. The synthesis of Ag and Ag@ZnO nanoparticles took place at similar conditions ensuring that the plain Ag particles have about the same size as the Ag cores, with diameters in the range of 3-4 $\mathrm{nm}$. For bare $\mathrm{Ag}$ nanoparticles in this size regime, it is known that the employed laser with a photon energy of $3.02 \mathrm{eV}(410 \mathrm{~nm})$ can excite localized surface plasmons [50,51]. Note that the exact position of the LSPR also depends on the substrate and is, thus, not exactly known for the prepared particles on ITO. For particles produced by He droplet synthesis on fused silica, however, a relatively broad LSPR was found with a maximum around $450 \mathrm{~nm}$ [29].

In photoelectron spectroscopy, the electrons originating from the Ag core have to pass the $\mathrm{ZnO}$ shell in order to reach the detector. However, in contrast to the UPS measurements, the inelastic mean free path of the electrons after excitation with the laser beam is up to several tens of $\mathrm{nm}$, which ensures that the acquired data contains information about the core material [45].

In order to compare the kinetic energy of the collected electrons, the work function $(\Phi)$ difference between the samples has to be accounted for. $\Phi$ was determined by intersecting the abscissa with a tangent through the turning point of the rising edge of the secondary electron peak in the collected spectra. In addition, the Schottky effect, which emerges due to the presence of the $12 \mathrm{kV}$ extractor field at a sample distance of $1.6 \mathrm{~mm}$, has been considered [52] and introduces a reduction of $\Phi$ by $\Delta E=104 \mathrm{meV}$. The obtained work functions correspond to $(3.7 \pm 0.2) \mathrm{eV}$ for $\mathrm{ZnO},(4.2 \pm 0.2) \mathrm{eV}$ for $\mathrm{Ag}$, and $(4.0 \pm 0.2) \mathrm{eV}$ for Ag@ZnO. Since these values exceed the photon energy of the laser, it can be assumed that the spectra plotted as a function of $E_{\text {kin }}$ in Fig. 5 correspond to electrons excited in a two-photon process with a maximum overall available energy of $6.04 \mathrm{eV}$ (at the Fermi cutoff). For a comparable graphic

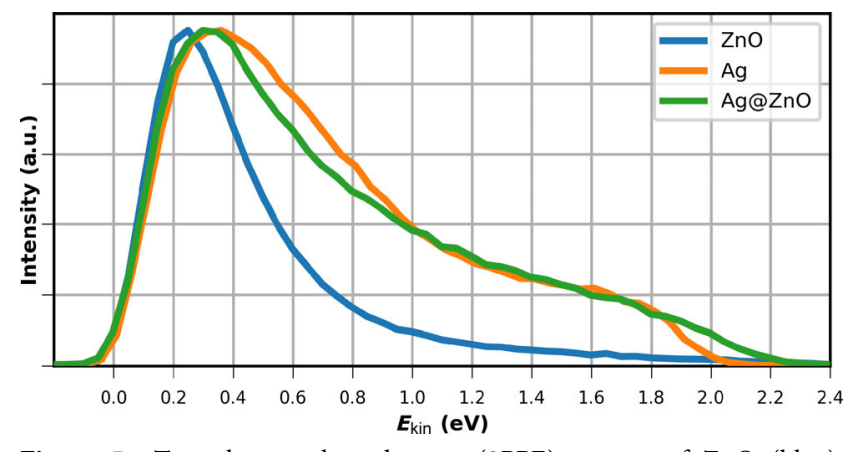

Figure 5 Two-photon photoelectron (2PPE) spectra of $\mathrm{ZnO}$ (blue), Ag (orange), and Ag@ZnO (green) nanoparticles on ITO irradiated with $3.02 \mathrm{eV}(410 \mathrm{~nm})$ p-polarized laser light. The significant increase of the electron yield at higher kinetic energies is attributed to an enhancement by the excitation of Ag surface plasmons.

presentation the signal intensities are normalized to the maxima of the secondary electron peaks of the spectra.

In Fig. 5 it can be seen that the 2PPE spectrum of $\mathrm{ZnO}$, depicted in blue, shows a prominent secondary electron peak that decays fast with increasing electron kinetic energy with only a very weak signal intensity towards the Fermi cutoff. However, in the case of the Ag spectrum (orange) a significant enhancement of electron yield at higher kinetic energies is observed. Such an increase in high energy photons has been attributed to an enhancement of the surface electric field by the excitation of plasmons in Ag nanoparticles [39, 53]. Intriguingly, the Ag@ZnO spectrum closely resembles the form of the plain Ag spectrum. Consequently, it is concluded that also in this case a localized surface plasmon is excited. The precise origin of the electrons, however, remains elusive. Plasmon excitation in the Ag core leads to an enhancement of the electric field close by the nanoparticle such that the photoelectrons may either be created in the Ag or the $\mathrm{ZnO}$ shell. Furthermore, in contrast to the plain Ag particles that were kept under UHV conditions, the Ag@ZnO particles were exposed to ambient air. The $\mathrm{ZnO}$ shells provide a protective layer preventing a contamination of the Ag cores with water or other hydrocarbons, while still preserving their plasmonic properties.

\section{Conclusions}

In the course of this study, we showed a novel way to produce high quality Ag@ZnO core@shell nanoparticles by employing the helium nanodroplet synthesis approach. Compared to other synthesis routes, this technique has the advantage of utilizing superfluid helium as pristine "nanolabs" in which the particles are grown without the addition of any solvents or other chemical agents. Thorough STEM investigations reveal the uniform and evenly distributed shape of the wurtzite $\mathrm{ZnO}$ shells surrounding the Ag cores, not only for spherically shaped particles but also for larger, wire-like core@shell structures. Thereby, the $\mathrm{ZnO}$ provides a very stable and reliable protective cover for the plasmonic Ag cores. This is indicated by $2 \mathrm{PPE}$ spectroscopy, where a significant plasmon enhancement of the photoelectron yield is observed for plain $\mathrm{Ag}$ as well as Ag@ZnO core@shell nanoparticles. Furthermore, the oxidation state of the $\mathrm{Zn} / \mathrm{ZnO}$ shell system was investigated by accessing the $\mathrm{Zn} 3 \mathrm{~d}$ core level with UPS, opening up possible routes for the synthesis and in situ monitoring of triple layer Ag@Zn@ZnO core@shell systems on the nanoscale. The stability of the Ag@ZnO nanoparticles and the preservation of important functional features upon exposure to ambient conditions sets the ground for possible future applications outside of the laboratory environment. 


\section{Acknowledgements}

The authors gratefully acknowledge support from the Austrian Science Fund (FWF) under grant P30940-N36 as well as support from NAWI Graz. Further funding for this research was received from the European Union's Horizon 2020 research program under Grant Agreement No. 823717-ESTEEM3. The authors thank Maximilian Lasserus, Roman Messner, and Martin Schnedlitz for productive discussions and helpful suggestions.

Funding note: Open access funding provided by Graz University of Technology.

Electronic Supplementary Material: Supplementary material (STEM and SEM images of Ag@ZnO nanoparticles in varying magnifications and additional information on the $\mathrm{ZnO}$ structure determination and the modality of the surface coverage estimation) is available in the online version of this article at https://doi.org/10.1007/s12274-020-2961-z.

Open Access This article is licensed under a Creative Commons Attribution 4.0 International License, which permits use, sharing, adaptation, distribution and reproduction in any medium or format, as long as you give appropriate credit to the original author(s) and the source, provide a link to the Creative Commons licence, and indicate if changes were made.

The images or other third party material in this article are included in the article's Creative Commons licence, unless indicated otherwise in a credit line to the material. If material is not included in the article's Creative Commons licence and your intended use is not permitted by statutory regulation or exceeds the permitted use, you will need to obtain permission directly from the copyright holder.

To view a copy of this licence, visit http://creativecommons.org/licenses/by/4.0/.

\section{References}

[1] Ong, C. B.; Ng, L. Y.; Mohammad, A. W. A review of $\mathrm{ZnO}$ nanoparticles as solar photocatalysts: Synthesis, mechanisms and applications. Renew. Sust. Energy Rev. 2018, 81, 536-551.

[2] Qi, K. H.; Cheng, B.; Yu, J. G.; Ho, W. Review on the improvement of the photocatalytic and antibacterial activities of $\mathrm{ZnO}$. J. Alloys Compd. 2017, 727, 792-820.

[3] Mishra, S. K.; Srivastava, R. K.; Prakash, S. G.; Yadav, R. S.; Panday, A. C. Photoluminescence and photoconductive characteristics of hydrothermally synthesized $\mathrm{ZnO}$ nanoparticles. Opto-Electron. Rev. 2010, 18, 467-473.

[4] Özgür, Ü.; Hofstetter, D.; Morkoç, H. ZnO devices and applications: A review of current status and future prospects. Proc. IEEE 2010, 98, 1255-1268.

[5] Wang, W. W.; Zhu, Y. J. Shape-controlled synthesis of zinc oxide by microwave heating using an imidazolium salt. Inorg. Chem. Commun. 2004, 7, 1003-1005.

[6] Kołodziejczak-Radzimska, A.; Jesionowski, T. Zinc oxide-from synthesis to application: A review. Materials 2014, 7, 2833-2881.

[7] Ghanbari Shohany, B.; Khorsand Zak, A. Doped ZnO nanostructures with selected elements - Structural, morphology and optical properties: A review. Ceram. Int. 2020, 46, 5507-5520.

[8] Georgekutty, R.; Seery, M. K.; Pillai, S. C. A highly efficient Ag-ZnO photocatalyst: Synthesis, properties, and mechanism. J. Phys. Chem. C 2008, 112, 13563-13570.

[9] Xiao, M. D.; Jiang, R. B.; Wang, F.; Fang, C. H.; Wang, J. F.; Yu, J. C. Plasmon-enhanced chemical reactions. J. Mater. Chem. A 2013, $1,5790-5805$.

[10] Gomez, L. F.; Loginov, E.; Sliter, R.; Vilesov, A. F. Sizes of large He droplets. J. Chem. Phys. 2011, 135, 154201.

[11] Gomez, L. F.; Ferguson, K. R.; Cryan, J. P.; Bacellar, C.; Tanyag, R. M. P.; Jones, C.; Schorb, S.; Anielski, D.; Belkacem, A.; Bernando,
C. et al. Shapes and vorticities of superfluid helium nanodroplets. Science 2014, 345, 906-909.

[12] Mozhayskiy, V.; Slipchenko, M. N.; Adamchuk, V. K.; Vilesov, A. F. Use of helium nanodroplets for assembly, transport, and surface deposition of large molecular and atomic clusters. J. Chem. Phys. 2007, 127, 094701.

[13] Boatwright, A.; Feng, C.; Spence, D.; Latimer, E.; Binns, C.; Ellis, A. M.; Yang, S. F. Helium droplets: A new route to nanoparticles. Faraday Discuss. 2013, 162, 113-124.

[14] Volk, A.; Thaler, P.; Koch, M.; Fisslthaler, E.; Grogger, W.; Ernst, W. E. High resolution electron microscopy of Ag-clusters in crystalline and non-crystalline morphologies grown inside superfluid helium nanodroplets. J. Chem. Phys. 2013, 138, 214312.

[15] Thaler, P.; Volk, A.; Knez, D.; Lackner, F.; Haberfehlner, G.; Steurer, J.; Schnedlitz, M.; Ernst, W. E. Synthesis of nanoparticles in helium droplets - A characterization comparing mass-spectra and electron microscopy data. J. Chem. Phys. 2015, 143, 134201.

[16] Toennies, J. P.; Vilesov, A. F. Superfluid helium droplets: A uniquely cold nanomatrix for molecules and molecular complexes. Angew. Chem., Int. Ed. 2004, 43, 2622-2648.

[17] Callegari, C.; Ernst, W. E. Helium Droplets as nanocryostats for molecular spectroscopy - from the vacuum ultraviolet to the microwave regime. In: Handbook of High-Resolution Spectroscopy. John Wiley \& Sons, Inc.: New York, 2011; pp 1551-1594.

[18] Yang, S. F.; Ellis, A. M.; Spence, D.; Feng, C.; Boatwright, A.; Latimer, E.; Binns, C. Growing metal nanoparticles in superfluid helium. Nanoscale 2013, 5, 11545-11553.

[19] Hauser, A. W.; Volk, A.; Thaler, P.; Ernst, W. E. Atomic collisions in suprafluid helium-nanodroplets: Timescales for metal-cluster formation derived from He-density functional theory. Phys. Chem. Chem. Phys. 2015, 17, 10805-10812.

[20] Volk, A.; Thaler, P.; Knez, D.; Hauser, A. W.; Steurer, J.; Grogger, W.; Hofer, F.; Ernst, W. E. The impact of doping rates on the morphologies of silver and gold nanowires grown in helium nanodroplets. Phys. Chem. Chem. Phys. 2016, 18, 1451-1459.

[21] Gomez, L. F.; Loginov, E.; Vilesov, A. F. Traces of vortices in superfluid helium droplets. Phys. Rev. Lett. 2012, 108, 155302.

[22] Gessner, O.; Vilesov, A. F. Imaging quantum vortices in superfluid helium droplets. Аnnu. Rev. Phys. Chem. 2019, 70, 173-198.

[23] Sergeev, Y. A.; Barenghi, C. F. Particles-vortex interactions and flow visualization in ${ }^{4}$ He. J. Low Temp. Phys. 2009, 157, 429.

[24] Gordon, E.; Karabulin, A.; Matyushenko, V.; Sizov, V.; Khodos, I. Stability and structure of nanowires grown from silver, copper and their alloys by laser ablation into superfluid helium. Phys. Chem. Chem. Phys. 2014, 16, 25229-25233.

[25] Schnedlitz, M.; Lasserus, M.; Knez, D.; Hauser, A. W.; Hofer, F.; Ernst, W. E. Thermally induced breakup of metallic nanowires: Experiment and theory. Phys. Chem. Chem. Phys. 2017, 19, 9402-9408.

[26] Lasserus, M.; Schnedlitz, M.; Knez, D.; Messner, R.; Schiffmann, A.; Lackner, F.; Hauser, A. W.; Hofer, F.; Ernst, W. E. Thermally induced alloying processes in a bimetallic system at the nanoscale: $\mathrm{AgAu}$ sub-5 $\mathrm{nm}$ core-shell particles studied at atomic resolution. Nanoscale 2018, 10, 2017-2024.

[27] Schiffmann, A.; Knez, D.; Lackner, F.; Lasserus, M.; Messner, R.; Schnedlitz, M.; Kothleitner, G.; Hofer, F.; Ernst, W. E. Ultra-thin h-BN substrates for nanoscale plasmon spectroscopy. J. Appl. Phys. 2019, 125, 023104.

[28] Thaler, P.; Volk, A.; Lackner, F.; Steurer, J.; Knez, D.; Grogger, W.; Hofer, F.; Ernst, W. E. Formation of bimetallic core-shell nanowires along vortices in superfluid He nanodroplets. Phys. Rev. B 2014, 90 , 155442.

[29] Lackner, F.; Schiffmann, A.; Lasserus, M.; Messner, R.; Schnedlitz, M.; Fitzek, H.; Pölt, P.; Knez, D.; Kothleitner, G.; Ernst, W. E. Helium nanodroplet assisted synthesis of bimetallic Ag@Au nanoparticles with tunable localized surface plasmon resonance. Eur. Phys. J. D 2019, 73, 104.

[30] Lasserus, M.; Knez, D.; Schnedlitz, M.; Hauser, A. W.; Hofer, F.; Ernst, W. E. On the passivation of iron particles at the nanoscale. Nanoscale Adv. 2019, 1, 2276-2283.

[31] Thaler, P.; Volk, A.; Ratschek, M.; Koch, M.; Ernst, W. E. Molecular dynamics simulation of the deposition process of cold Ag-clusters under different landing conditions. J. Chem. Phys. 2014, 140, 044326. 
[32] de Lara-Castells, M. P.; Aguirre, N. F.; Stoll, H.; Mitrushchenkov, A. O.; Mateo, D.; Pi, M. Communication: Unraveling the ${ }^{4} \mathrm{He}$ dropletmediated soft-landing from ab initio-assisted and time-resolved density functional simulations: $\mathrm{Au} @{ }^{4} \mathrm{He}_{300} / \mathrm{TiO}_{2}(110)$. J. Chem. Phys. 2015, 142, 131101.

[33] Haberfehlner, G.; Thaler, P.; Knez, D.; Volk, A.; Hofer, F.; Ernst, W. E.; Kothleitner, G. Formation of bimetallic clusters in superfluid helium nanodroplets analysed by atomic resolution electron tomography. Nat. Commun. 2015, 6, 8779.

[34] He, M.; Protesescu, L.; Caputo, R.; Krumeich, F.; Kovalenko, M. V. A general synthesis strategy for monodisperse metallic and metalloid nanoparticles (In, $\mathrm{Ga}, \mathrm{Bi}, \mathrm{Sb}, \mathrm{Zn}, \mathrm{Cu}, \mathrm{Sn}$, and their alloys) via in situ formed metal long-chain amides. Chem. Mater. 2015, 27, 635-647.

[35] Walther, T.; Humphreys, C. J. A quantitative study of compositional profiles of chemical vapour-deposited strained silicon-germanium/ silicon layers by transmission electron microscopy. J. Cryst. Growth 1999, 197, 113-128.

[36] Schreyer, M.; Guo, L.; Thirunahari, S.; Gao, F.; Garland, M. Simultaneous determination of several crystal structures from powder mixtures: The combination of powder X-ray diffraction, band-target entropy minimization and Rietveld methods. J. Appl. Cryst. 2014, 47, 659-667.

[37] Prieur, D.; Bonani, W.; Popa, K.; Walter, O.; Kriegsman, K. W.; Engelhard, M. H.; Guo, X. F.; Eloirdi, R.; Gouder, T.; Beck, A. et al. Size dependence of lattice parameter and electronic structure in $\mathrm{CeO}_{2}$ nanoparticles. Inorg. Chem. 2020, 59, 5760-5767.

[38] Schindelin, J.; Arganda-Carreras, I.; Frise, E.; Kaynig, V.; Longair, M.; Pietzsch, T.; Preibisch, S.; Rueden, C.; Saalfeld, S.; Schmid, B. et al. Fiji: An open-source platform for biological-image analysis. Nat. Methods 2012, 9, 676-682.

[39] Lehmann, J.; Merschdorf, M.; Pfeiffer, W.; Thon, A.; Voll, S.; Gerber, G. Surface plasmon dynamics in silver nanoparticles studied by femtosecond time-resolved photoemission. Phys. Rev. Lett. 2000, 85, 2921-2924.

[40] Evans, S. Energy calibration secondary standards for X-ray photoelectron spectrometers. Surf. Interface Anal. 1985, 7, 299-302.

[41] Gaarenstroom, S. W.; Winograd, N. Initial and final state effects in the ESCA spectra of cadmium and silver oxides. J. Chem. Phys. 1977, $67,3500-3506$.
[42] Ley, L.; Kowalczyk, S. P.; McFeely, F. R.; Pollak, R. A.; Shirley, D. A. X-Ray photoemission from zinc: Evidence for extra-atomic relaxation via semilocalized excitons. Phys. Rev. B 1973, 8, 23922402 .

[43] Schön, G. Auger and direct electron spectra in X-ray photoelectron studies of zinc, zinc oxide, gallium and gallium oxide. J. Electron Spectrosc. Relat. Phenom. 1973, 2, 75-86.

[44] Gutmann, S.; Conrad, M.; Wolak, M. A.; Beerbom, M. M.; Schlaf, R. Work function measurements on nano-crystalline zinc oxide surfaces. J. Appl. Phys. 2012, 111, 123710.

[45] Seah, M. P.; Dench, W. A. Quantitative electron spectroscopy of surfaces: A standard data base for electron inelastic mean free paths in solids. Surf. Interface Anal. 1979, 1, 2-11.

[46] Misra, M.; Kapur, P.; Nayak, M. K.; Singla, M. Synthesis and visible photocatalytic activities of a Au@Ag@ZnO triple layer core-shell nanostructure. New J. Chem. 2014, 38, 4197-4203.

[47] Aranishi, K.; Jiang, H. L.; Akita, T.; Haruta, M.; Xu, Q. One-step synthesis of magnetically recyclable $\mathrm{Au} / \mathrm{Co} / \mathrm{Fe}$ triple-layered coreshell nanoparticles as highly efficient catalysts for the hydrolytic dehydrogenation of ammonia borane. Nano Res. 2011, 4, 1233-1241.

[48] Mazumder, V.; Chi, M. F.; More, K. L.; Sun, S. H. Synthesis and characterization of multimetallic $\mathrm{Pd} / \mathrm{Au}$ and $\mathrm{Pd} / \mathrm{Au} / \mathrm{FePt}$ core/shell nanoparticles. Angew. Chem., Int. Ed. 2010, 49, 9368-9372.

[49] LaGrow, A. P.; Lloyd, D. C.; Gai, P. L.; Boyes, E. D. In situ scanning transmission electron microscopy of $\mathrm{Ni}$ nanoparticle redispersion via the reduction of hollow NiO. Chem. Mater. 2018, 30, 197-203.

[50] Munzinger, M.; Wiemann, C.; Rohmer, M.; Guo, L.; Aeschlimann, M.; Bauer, M. The lateral photoemission distribution from a defined cluster/substrate system as probed by photoemission electron microscopy. New J. Phys. 2005, 7, 68.

[51] Rohmer, M.; Ghaleh, F.; Aeschlimann, M.; Bauer, M.; Hövel, H. Mapping the femtosecond dynamics of supported clusters with nanometer resolution. Eur. Phys. J. D 2007, 45, 491-499.

[52] Knapp, A. G. Surface potentials and their measurement by the diode method. Surf. Sci. 1973, 34, 289-316.

[53] Evers, F.; Rakete, C.; Watanabe, K.; Menzel, D.; Freund, H. J. Twophoton photoemission from silver nanoparticles on thin alumina films: Role of plasmon excitation. Surf. Sci. 2005, 593, 43-48. 